\title{
Size Doesn't Matter: Scaffold Diversity, Shape Diversity and Biological Activity of Combinatorial Libraries
}

\author{
Wolfgang H.B. Sauer and Matthias K. Schwarz*
}

\begin{abstract}
Among questions of significant interest to the pharmaceutical industry are the relative merits of screening numerous libraries of moderate size $(<5000$ members) comprising many different scaffold structures, versus screening numerically larger libraries (>100,000 members) of a limited selection of chemotypes. Using a newly developed computational method to assess the diversity in molecular shape associated with different compound sets, we have shown that single-scaffold libraries, irrespective of their size, are restricted to a limited range of molecular shapes, whereas collections of several small libraries around distinct chemical scaffolds can produce a higher degree of shape diversity. A comparison of the molecular shape distribution patterns associated with different MDDR (MDL ${ }^{\circledR}$ Drug Data Report) subsets of known biological activities corroborates the intuitive notion that molecular shape is intimately linked to biological activity, and that a high degree of shape (hence scaffold) diversity in screening collections will increase the odds of addressing a broad range of biological targets. In order to cope with the challenge of assembling a portfolio of small libraries around various central scaffolds in a reasonable amount of time, the combinatorial chemist must now, more than ever, seek to optimize the synthetic outcome with respect to the efforts in terms of chemistry set-up and validation.
\end{abstract}

Keywords: Biological activity $\cdot$ Combinatorial library $\cdot$ Molecular shape $\cdot$ Scaffold $\cdot$ Shape triangle diagram

\section{Introduction}

Viewed by many as a mere scientific curiosity upon its introduction in the early 90s, combinatorial chemistry has now established itself as one of the key tools in the modern pharmaceutical drug discovery environment. Combinatorial chemistry enables the rapid simultaneous synthesis of large compound collections ('libraries'), by

${ }^{*}$ Correspondence: Dr. M.K. Schwarz Serono Pharmaceutical Research Institute Department of Chemistry

14, Chemin des Aulx

CH-1228 Plan-les-Ouates (Geneva)

Tel.: +41227069820

Fax: +4122 7946965

E-Mail: Matthias.Schwarz@Serono.com bringing together sets of reactive monomers ('building blocks'), $\mathrm{A}_{1}, \mathrm{~A}_{2} \ldots \mathrm{A}_{\mathrm{m}} ; \mathrm{B}_{1}, \mathrm{~B}_{2}, \ldots$ $\mathrm{B}_{\mathrm{n}} ; \cdots \mathrm{Z}_{1}, \mathrm{Z}_{2} \ldots \mathrm{Z}_{\mathrm{p}}$, in such a way that in principle all combinations of final compounds of general structure AB. . Z Z are formed. Typically, all compounds of a given library will share a common structural moiety, referred to as the 'template', 'scaffold', or 'chemotype' [1], which can either be a constant central building block or a functional group generated by the coupling chemistry between the different building blocks. Experience has shown that the ratelimiting step in library generation is the validation and optimization of the chemistry associated with a particular scaffold structure, and the demonstration of its compatibility with a range of diverse peripheral building blocks having different chemical reactivities. Once the chemical protocol is worked out, the actual production of the library is usually just a matter of weeks, virtually irrespective of the library size. This means that the generation of one large library of 50,000 members involves a significantly smaller investment in terms of time and manpower than the assembly of a portfolio of ten 5000 member libraries around ten different central scaffolds. Remarkably though, whilst the early days of combinatorial chemistry were dominated by very large libraries ( $>10,000$ members), the current trend in the pharmaceutical industry clearly goes towards collections of relatively small libraries (around 1000 members) comprising many different chemotypes. Arguably, practicality and feasibility arguments have been superseded by considerations related to the biological activity potential of the first-generation combinatorial libraries. A common (yet rarely publicized) observation in high-throughput screening of early combinatorial libraries against a number of biological targets was that, for a particular scaffold, the biological results tended to be 'sporadic', i.e. the hit rate throughout the different targets was either very high or essentially zero. On the other hand, compound sets containing a wealth of different chemotypes, such as natural product or commercial compound collections, tended to exhibit more consistent hit rates across a variety of targets. These observations led us and others [2] to 
speculate that a combinatorial library derived from a single scaffold, irrespective of the library size, could innately not become diverse enough to be able to interact with a number of different biological targets. In order to formally verify this hypothesis, we have recently carried out a series of studies in our laboratory [3]. The present account summarizes the results, which indeed indicate that small multiple-scaffold libraries are superior to large single-scaffold libraries in terms of their bio-relevance, i.e. their potential to hit a broad panel of biological targets.

\section{Assessing the Diversity of Combinatorial Libraries: The Choice of the 'Right' Descriptors}

In order to be able to compare different combinatorial libraries in terms of their biorelevant diversity, i.e. their potential to produce hits against a wide range of biological targets, we first had to define which molecular properties or descriptors the diversity analysis should be based upon. From the extensive literature published on the antipodal concepts of diversity (or dissimilarity) and similarity (see [3]), one can safely conclude that a generally applicable 'default set' of molecular descriptors is currently not available (and probably does not exist) [4]. Since every particular set of descriptors defines a unique chemical space with an idiosyncratic distribution of the compounds to be compared, the choice of the 'right' descriptors will usually be governed by the nature of the questions to be answered. We reasoned that for the purpose of our study, a molecular descriptor or property needed to be

(i) a priori correlated with, and predictive for, biological activity,

(ii) (back-) translatable into chemical structure terms, and

(iii)fast to calculate.

With this in mind, we decided to choose molecular shape as the basis for our diversity analysis. Three-dimensional molecular shape intuitively meets the criterion of a descriptor being a priori correlated with, and predictive for, biological activity, since a compound will only modulate the activity of a biological target, if it matches the appropriate cavities presented by the biological counterpart. To the chemist, molecular shape is an intelligible descriptor that can be rationalized and, if needed, modified in a predictable way. The time needed to compute the molecular shape of a particular compound will depend on the way in which the shape information is captured and represented (for a summary of shape descrip- tors, see [3]). As a compromise between complexity and information content of the representation, we decided to evaluate normalized ratios of principal moments of inertia (PMI) for their potential to serve as an intuitive, albeit rather crude, way to describe molecular shape. Thus, for each compound, the Corina 3D-structure [5][6] was used to calculate the three principal moments of inertia, sorted by ascending magnitude $\mathbf{I}_{1}, \mathbf{I}_{2}$, and $\mathbf{I}_{3}$. Subsequently, normalization was performed by dividing the two lower PMI values $\left(\mathbf{I}_{1}\right.$ and $\left.\mathbf{I}_{2}\right)$ by the highest value $\left(\mathbf{I}_{3}\right)$, generating two characteristic values of normalized PMI ratios (NPRs) for each compound ( $\mathbf{I}_{1} / \mathbf{I}_{3}$ and $\left.\mathbf{I}_{2} / \mathbf{I}_{3}\right)$. When finally plotted against each other, the resulting graph, shown in Fig. 1, represents an isosceles triangle, into which all compounds are projected. It is defined by its three corners, wherein the vector $\left[\mathbf{I}_{1} / \mathbf{I}_{3}\right.$, $\left.\mathbf{I}_{2} / \mathbf{I}_{3}\right]$ equals [1,1], [0.5,0.5], and [0,1], corresponding to archetype 'envelope' shapes of, respectively, spheres, discs, and rods. Some examples for molecules exhibiting these extremes, as well as intermediate geometries are shown in Fig. 1.

Using this computational approach, the range of molecular shapes associated with a given compound set can rapidly be assessed based on the corresponding distribution pattern generated by plotting the NPRs of all compounds into the triangle plot. This way, inter-set similarity or dissimilarity in terms of molecular shape can usually be appreciated already by mere visual inspection of the respective triangle diagrams. To eliminate any potential subjective bias, the computational method was further developed to allow for a quantitative analy-

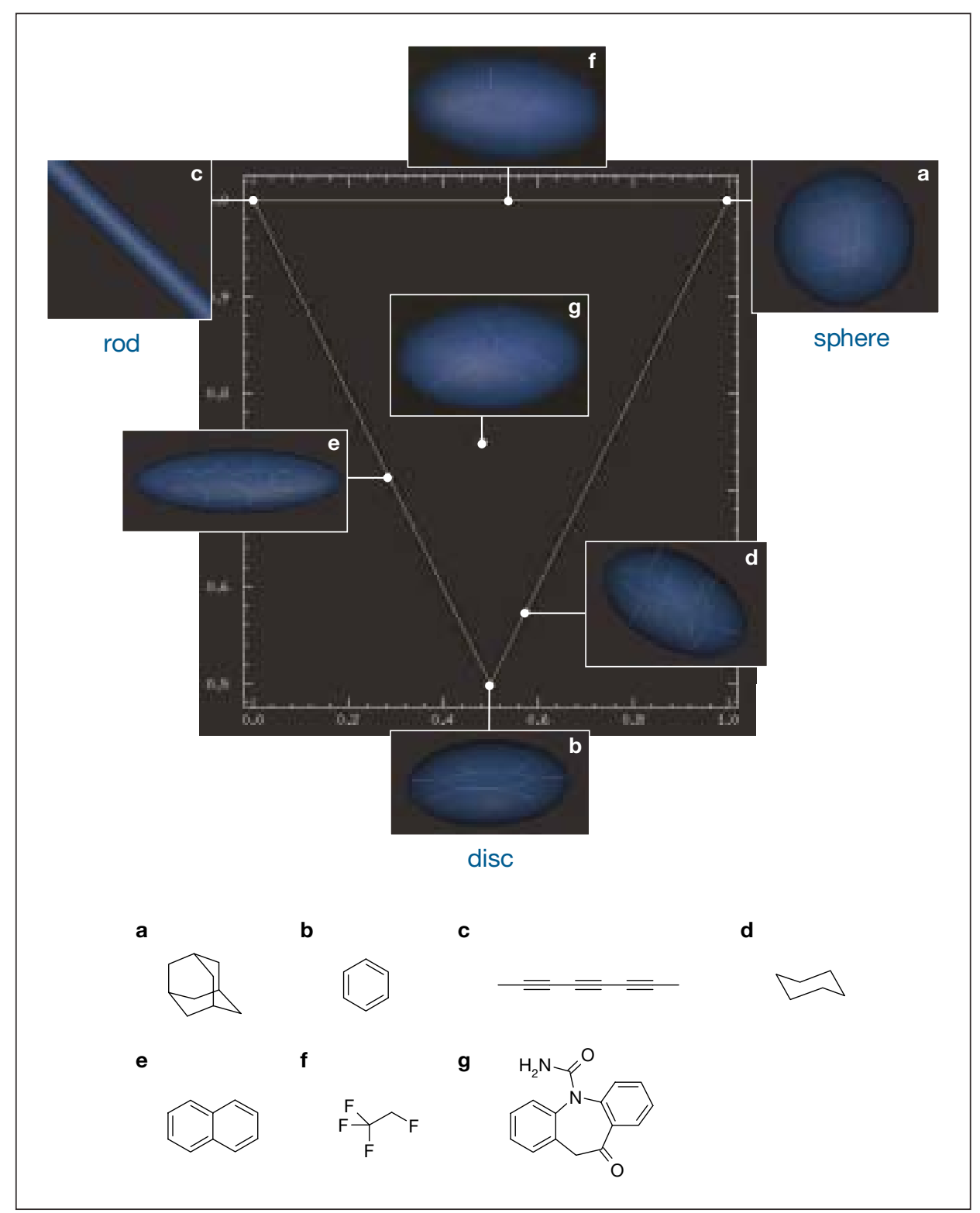

Fig. 1. Normalized PMI Ratios (NPRs) as shape descriptors: the position within the triangle reveals the 'envelope shape'. 
sis of the molecular shape similarity between different compound libraries. To this end, the triangle area was subdivided into 2500 triangles of equal size, followed by calculation of the corresponding membership counts for each library. These were then used to compute pair-wise indices as measures of inter-set similarity, in analogy to $e . g$. the Carbo indices traditionally used to quantify the similarity between pairs of molecules (for details, see [3]).

\section{Shape Diversity of Single- versus Multi-scaffold Combinatorial Libraries: A Model Case}

With these computational tools in hand, we turned our attention to devising an appropriate chemical model case related to the initial question about the relative merits of multiple-scaffold versus single-scaffold libraries in terms of their biorelevant diversity. At first sight, it appears trivial that a collection of libraries around different scaffolds will give rise to a higher shape diversity than a library built around a single central core, because the preset 3D geometry of a given scaffold restrains the number of possible spatial arrangements of the peripheral substituents, hence limits the number of attainable molecular shapes. One may, however, ask the question as to whether the apparent lack of diversity in terms of accessible shapes due to the fixed geometry of one particular scaffold can potentially be compensated by simply increasing the number of peripheral building blocks, that is by making a single-scaffold library sufficiently large. To investigate this, we took the example of a benzodiazepine scaffold with three positions for peripheral substituents. For the sake of simplicity, all three substituents were chosen from the same set of 13 diverse R-groups, leading to a relatively small library of 2197 members (A). This library was then enlarged to $>100,000$ members in two ways: (i) by increasing the set of peripheral substituents from 13 to 50 (while main- taining the same benzodiazepine scaffold), and

(ii) by increasing the number of central scaffolds from 1 to 50 (while keeping the initial set of 13 peripheral substituents), resulting in two big libraries, (B) and (C), of 125,000 and 109,850 members, respectively (for details on the choice and nature of peripheral substituents and scaffolds, see [3]).

The qualitative and quantitative shape diversity analysis of these three libraries reveals the following (see Fig. 2): The compounds belonging to library (A) are scattered in an intermediate region of the triangle graph, indicative for composite molecular envelope shapes with some spherical, some elongated and some discoid character. Notably, some areas, such as a strip along the left-hand triangle edge, remain unpopulated, pointing to the conspicuous absence of elongated discoid shapes. When the library size is multiplied by a factor of $>50$ by increasing the number of peripheral substituents, such as in library $(\mathbf{B})$, the global

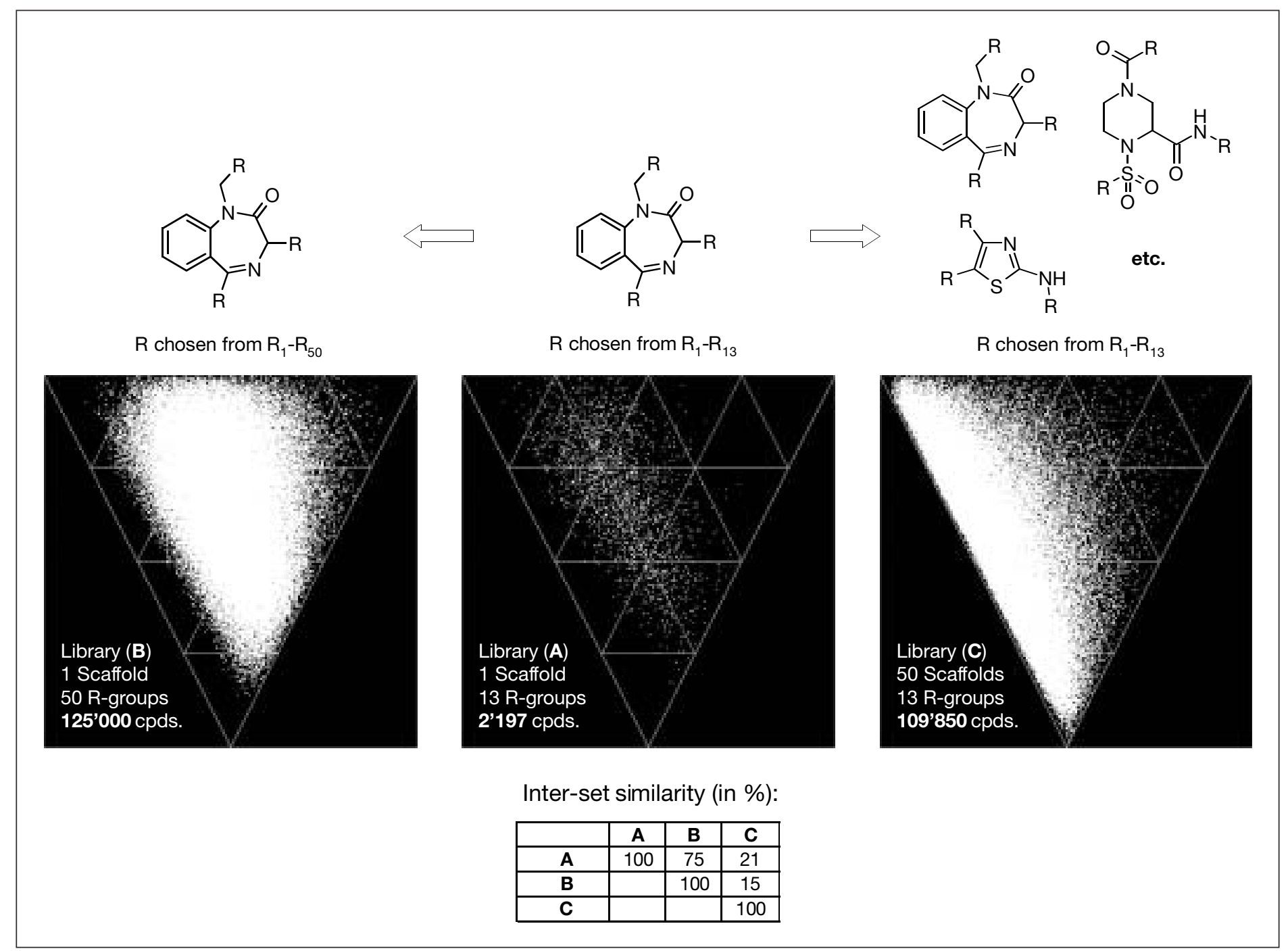

Fig. 2. Comparison of libraries (A), (B), and (C) based on shape diversity. 
picture remains the same, as shown by the high inter-set similarity index between (A) and $(\mathbf{B})$ of $75 \%$. Importantly, the left-hand strip remains entirely vacant, suggesting that, based on this specific benzodiazepine scaffold, elongated flat molecular shapes can intrinsically not be accessed, regardless of the library size. This result is instructive with respect to the initially mentioned sporadic ('all-or-nothing') hit rates often observed with early single-scaffold combinatorial libraries: for biological targets having cavities that will accommodate only flat elongated molecular shapes, the chances of finding a hit with this particular benzodiazepine scaffold are essentially zero, irrespective of the library size. Conversely, for targets requiring more globular shapes for optimal interaction, corresponding to the densely populated area of the triangle diagram, the likelihood of identifying hits from a collection like library $(\mathbf{B})$ is significant. Remarkably, if the single-scaffold library (A) is extended to a multi-scaffold collection of small libraries, such as library (C), a nearly complete ( $>80 \%$ ) coverage of the shape triangle diagram is observed, despite a slightly lower total number of compounds compared to library (B). In particular the stretch along the left-hand edge, found to be 'inaccessible' to the benzodiazepine library (B), is now densely populated in the case of library $(\mathbf{C})$, indicating a high incidence of elongated flat shapes. The drastic change in the molecular shape distribution associated with the transition from library (A) to library (C) is further illustrated by a low inter-set similarity index of $21 \%$. In aggregate, these results indicate that multi-scaffold libraries are indeed more diverse in terms of molecular shape than single-scaffold libraries of comparable size.

\section{Shape Diversity of Multi-scaffold Combinatorial Libraries: Minimizing the Redundancy}

The dense distribution observed in the left-hand part of the triangle diagram generated by library (C) (see Fig. 2) points to a possible redundancy between some of the 50 sub-libraries in terms of their molecular shape distributions, implying that the number of scaffolds may be significantly reduced, without impairing the overall coverage of the shape diagram. The quantitative inter-set shape similarity analysis offers a tool to prioritize different sublibraries against each other, based on their relative contributions to the overall shape diversity of a collection. From a practical point of view, this information is of sub- stantial value, considering that the time spent on library generation tends to increase proportionally with the number of different scaffold chemistries, as mentioned in the introductory remarks. Assuming, for example, that the benzimidazole library $\mathbf{S 5}$ was already part of an existing set, and a new library had to be selected for the next synthetic cycle amongst the four candidates S6, S10, S17, and $\mathbf{S 4 0}$, shown in Fig. 3, the shape analysis would suggest to prioritize S17 and S40, because S6 and S10 appear highly redundant with $\mathbf{S 5}$ in terms of their shape distribution. This example may seem trivial, since the recommendation derived from the shape diversity analysis (gratifyingly) coincides with what every chemist would intuitively propose by simply looking at the 2D-structures of the three benz- imidazole scaffolds S5, S6 and S10. In most cases, however, the shape similarity or dissimilarity between different libraries is difficult to appreciate based solely on the 2D-structures of the respective central scaffolds. Thus, the compounds built around the benzimidazolone scaffold $\mathbf{S 4 7}$, seemingly akin to the benzimidazole compounds of library S5 in 2D-structural terms, are found to differ from the latter quite substantially (similarity index $=44 \%$ ), in that their molecular envelope shapes display a higher discoid, and less elongated character (see Fig. 3). A posteriori, this observation can be understood, as the benzimidazolone scaffold $\mathbf{S 4 7}$, in contrast to the benzimidazole variant S5, orients one substituent away from the principal molecular rotation axis, thereby increasing the molecular extension

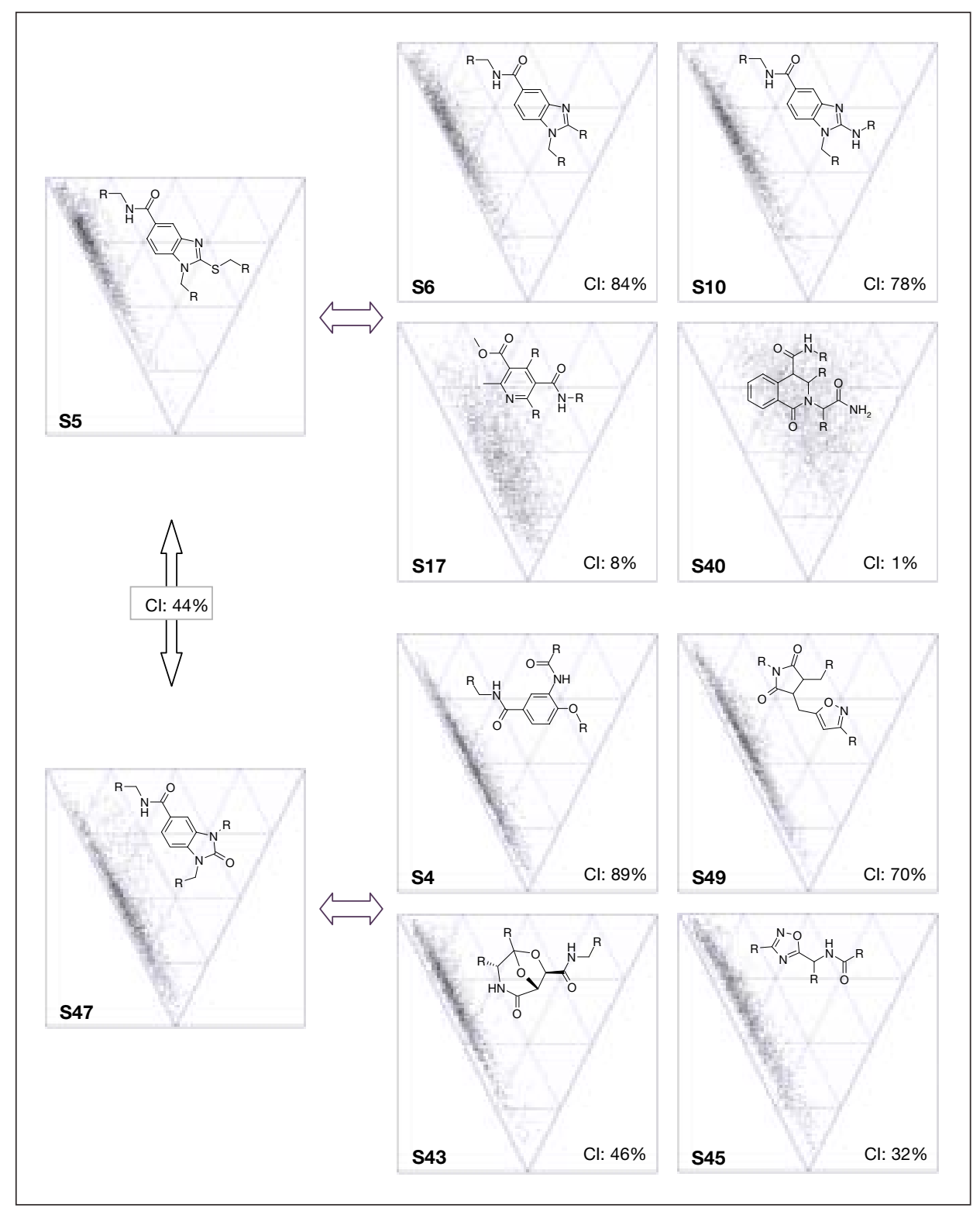

Fig. 3. Similarity analysis of combinatorial libraries with respect to shape space coverage and prioritization based on minimal inter-set redundancy (Cl: Carbo similarity index (in \%) of pair-wise comparisons). 
into the second dimension, and thus the discoid character of the corresponding compounds. Finally, the degree of shape similarity between library $\mathbf{S 4 7}$ and the group consisting of $\mathbf{S 4}, \mathbf{S 4 3}, \mathbf{S 4 5}$, and $\mathbf{S 4 9}$ is difficult to assess based on either the comparison of the 2D-structures or the visual inspection of the distribution patterns within the respective triangle diagrams. The quantitative inter-set similarity analysis, however, reveals quite clearly that the shape space coverage of $\mathbf{S 4 7}$ is most similar to $\mathbf{S 4}$, and most dissimilar to that of $\mathbf{S 4 5}$, which would then suggest to prioritize $\mathbf{S 4 5}$ over $\mathbf{S} 4$ in the context of library construction, if $\mathbf{S 4 7}$ was already part of the existing set.

It is important to note, that such recommendations can, at best, have the character of a first, crude filtering tool that should be used merely to prioritize between the different sub-libraries awaiting synthesis, but never to exclude any of them from eventually being made. In our opinion, the envelope shape of a molecule - together with its size - constitutes the first, most basic, level in a hierarchy of molecular descriptors [7], defining merely the playground for a wealth of secondary descriptors, such as polar surface area, hydrogenbonding surface potential, surface charge distribution, or presence and location of specific pharmacophoric elements. Shape complementarity is necessary, but alone not sufficient, for a compound to interact productively with a target. As an extreme example, one might invoke the case of $\mathrm{CH}_{4}$, $\mathrm{BH}_{4}^{-}$and $\mathrm{NH}_{4}^{+}$, which all have got the same shape (spherical), but entirely different electrostatic properties, making them prone to have different activities despite their identical shape.

\section{Shape Diversity and Biological Activity}

All data presented so far underline the paramount importance of the scaffold in defining the obtainable molecular shape range of a given combinatorial library and strongly advise the inclusion of as many different scaffolds as possible when assembling a compound collection aimed at maximum shape diversity. What has hitherto remained less well defined, however, is the link between shape diversity and biological activity, despite the intuitive notion that shape complementarity between small molecule and target is required for productive interaction (lock-and-key principle [8]). The single-scaffold benzodiazepine library (B) differs from the multi-scaffold library (C) in terms of molecular shape, in that it does not contain any compounds with markedly discoid and elongated shape (see Fig. 2). However, considering the remarkable pharmaceutical track record of benzodiazepines, one may rightfully ask whether the empty area in the triangle diagram of library (B) is biologically (or pharmacologically) relevant, that is whether the additional shape range accessible to library $(\mathbf{C})$ is prone to translate also into additional biological activities. To investigate this, we applied the shape diversity analysis to a collection of compounds known to interact with biological targets, namely the MDL Drug Data Report (MDDR; MDL Information Systems Inc., San Leandro, CA 94577, USA), comprising 101,800 compounds currently in development as potential drugs. The resulting graph, shown in Fig. 4, reveals a considerable proportion of the MDDR compounds to be scattered within the criti- cal area along the left-hand triangle side, demonstrating that flat elongated molecular shapes are by no means incompatible with biological activity, rather on the contrary. The remarkable similarity of the plots derived from library $(\mathbf{C})$ and MDDR gives an insight into why compound collections containing multiple chemotypes have frequently been found to outperform much larger (but single-scaffold) combinatorial libraries in terms of their average hit-rates against different biological targets.

Since many of the compounds listed in MDDR are annotated with their mechanism of action on molecularly defined biological targets, we decided to investigate to which extent similar biological activity would correlate with similar molecular shape. To this end, we extracted a number of activity classes from MDDR consisting of at least 250 compounds with known biological targets, and submitted them to the molecular envelope shape analysis. A selection of the resulting shape triangle diagrams, along with the corresponding pairwise Carbo similarity indices (in \%), is shown in Fig. 5 (for the complete dataset, see [3]). Inspection of the different graphs reveals first that the compounds of a particular activity class cluster in fairly localized areas of the triangle, which is remarkable in view of the fact that they typically comprise a number of distinct chemical series (or scaffolds). This would indeed suggest that each of these biological targets contains one major, characteristically shaped, receptive site that will single out those (and only those) compounds with complementary molecular shapes. Consequently, when assembling focused screening sets for a known biological target, it may be advisable to give prefer-

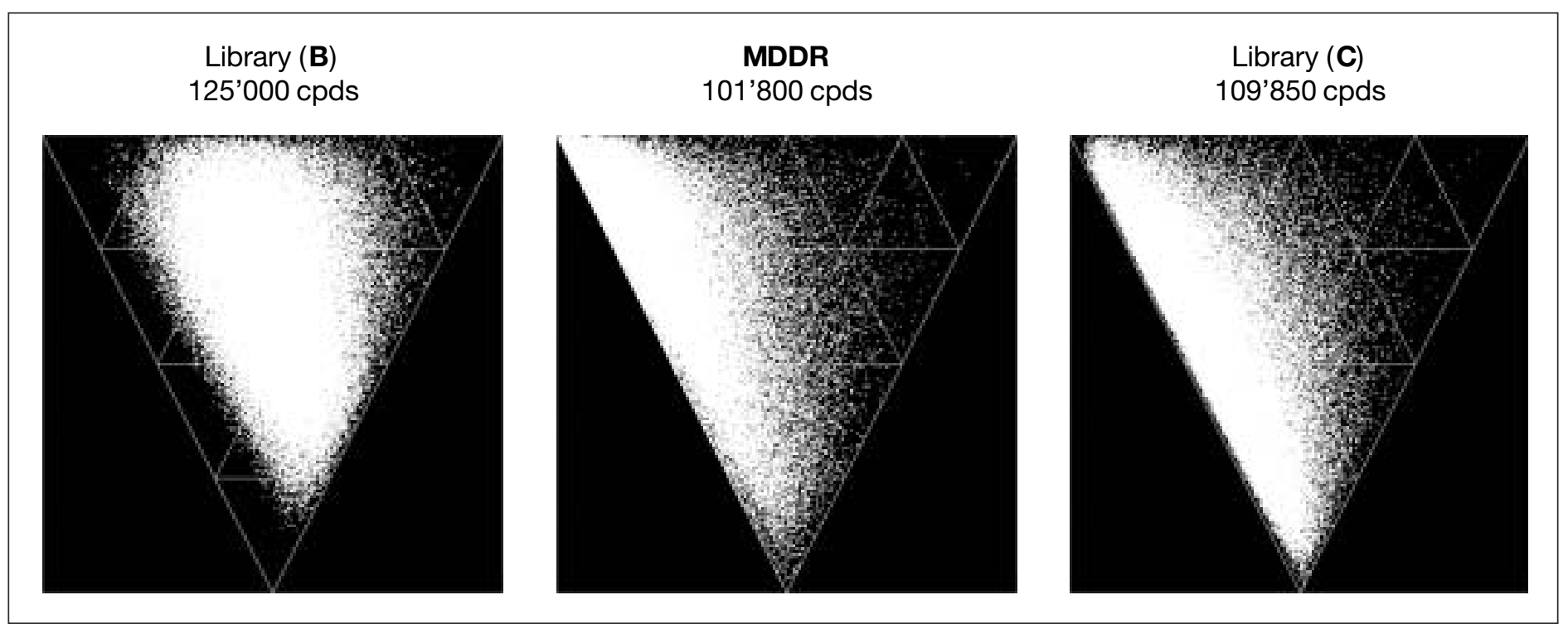

Fig. 4. Pharmacological relevance of complete shape triangle coverage: comparison of single-scaffold library (B), multi-scaffold library (C), and MDDR. 
ence to compounds that scatter in a similar area of the triangle diagram as compounds previously described to be active on this particular target. Other cases do exist, where the compounds active on a particular target are spread over a larger area of the triangle, often indicating the presence of different binding sites, a prime example being the Ca-channel blockers (data not shown, but see [3]). A second interesting observation is that compound sets $(06235,07701,07710)$ interacting with closely related biological targets, such as biogenic amine GPCRs (G-protein coupled receptors), show an inter-set similarity much above average (see Fig. 5). Notably, this strong similarity does not universally apply to all compound sets interacting with GPCRs. There seems to be a strong discrimination between GPCR subclasses based on the nature of their endogenous ligands (biogenic amines vs. peptides), as illustrated by the strikingly low similarities between the sets containing serotoninergic and dopaminergic ligands (06235, 07701, 07710) versus those consisting of e.g. $\mathrm{AT}_{1}$-antagonists (31432). As a final observation, the examples of enzyme inhibitors cited in Fig. 5 (52500, 78348, $78418,78454)$ tend to spread in regions again distinct from those occupied by the GPCR ligands (06235, 07701, 07710, 31432). This is, however, by no means a universally applicable rule, as shown by the case of the PLA2-inhibitors (78348), which are found to have a fairly similar shape distribution as the biogenic amine GPCR ligands (06235, 07701, 07710).

Taken together, the results presented above corroborate the notion that molecular shape is correlated with biological activity, and that a high degree of shape (hence scaffold) diversity in screening collections will increase the odds of addressing a broad range of biological targets. Clearly, one can cite numerous exceptions, in which chemical series with different shapes produce similar biological activities, either by interacting in a different way with the same site or by addressing different sites on the same biological target. It is noteworthy, however, that these exceptions do not contradict the basic thesis of striving for maximum shape diversity in primary screening collections, because it is highly desirable to identify several distinct chemical series active on a given target, to preempt potential downstream issues often encountered with one chemical family, such as toxicity or poor pharmacokinetics.

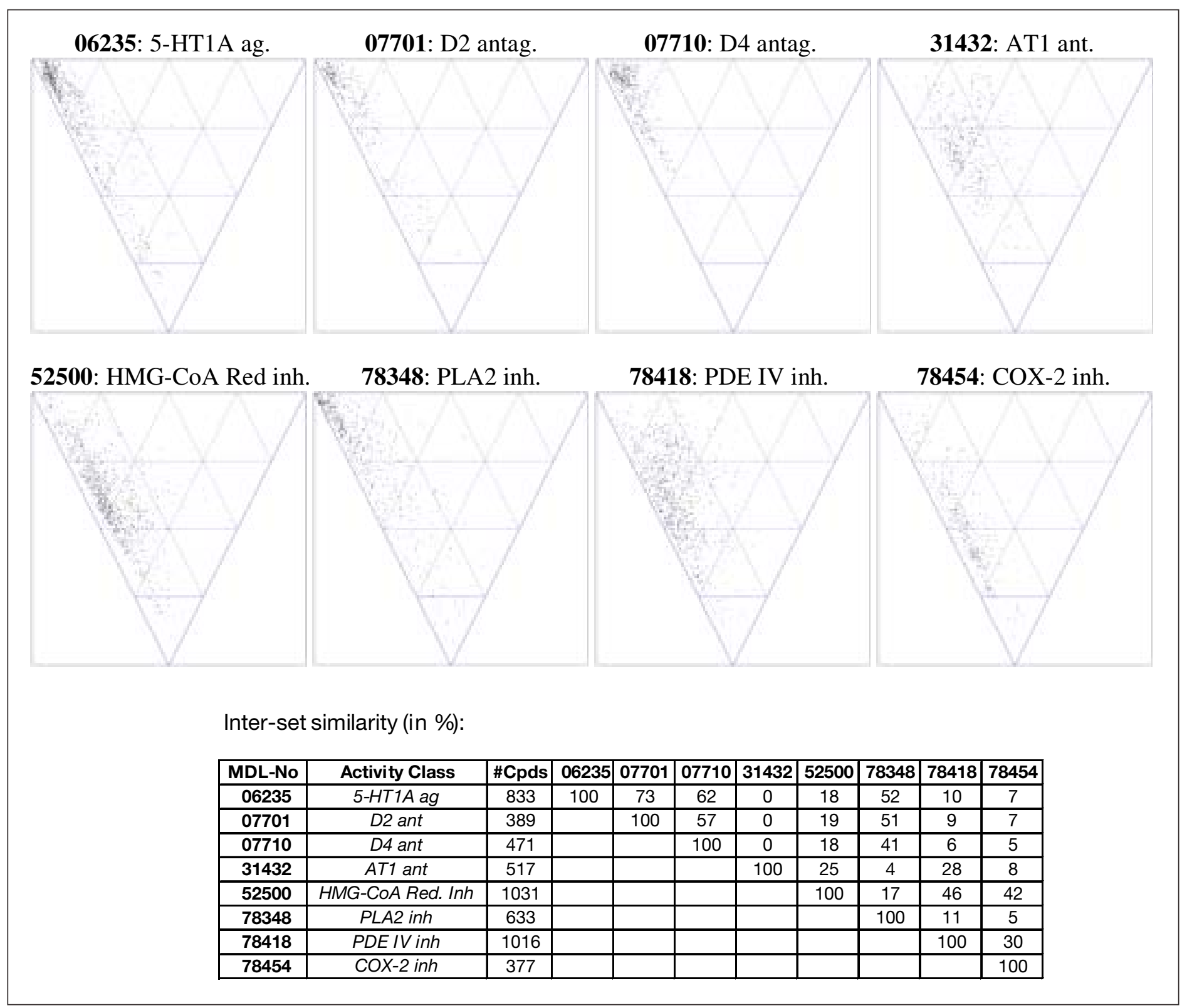

Fig. 5. Shape triangle diagrams of MDDR subsets grouped by biological activities. 


\section{Combinatorial Chemistry in 2003: How to Cope with the Challenge?}

The first-generation combinatorial libraries, despite their impressive size, have failed to provide the medicinal chemist with the plethora of new hits initially predicted by many commentators on the basis of the typical average hit rates obtained from screening of classical compound collections. This report offers one possible explanation in showing that the compounds of a combinatorial library built around one particular scaffold are insufficiently diverse in terms of molecular shape, which, in turn, is a key molecular property determining biological activity. As a consequence, the modern combinatorial chemist should strive to assemble a portfolio of small libraries around various central scaffolds, which represents a formidable challenge, as the time and effort spent on library generation tend to increase proportionally with the number of different scaffold chemistries. With this in mind, it is attractive to consider synthetic strategies that provide access to multiple, structurally diverse core structures starting from common, fundamental intermediates (synthons) and to use similar sequences of synthetic transformations in conjunction with the appropriate monomer inputs. This way, the synthetic protocols, once developed and optimized, can subsequently be re-used in different contexts to produce an array of different compound classes. Thus, the initial investment in establishing the practicability of the first scaffold chemistry will impact on the requisite time periods for subsequent chemistry development which will be successively shorter every time a new core structure synthesis is attempted. One example of such a versatile synthon is 4-fluoro-3-nitrobenzoic acid (Scheme 1). We and other groups have previously shown that a variety of benzofused heterocycles can be derived from resin-bound 4-fluoro-3-nitrobenzoic acid via solid-phase nucleophilic aromatic substitution $\left(\mathrm{S}_{N} \mathrm{Ar}\right)$ reactions with either sulfur- or nitrogen- nucleophiles. In addition, replacement of the aryl with heteroaryl cores, such as thiophene, affords the corresponding heteroaryl-, e.g. thieno-, fused scaffold structures, while variation of the attachment point to the resin opens access to the corresponding analogues lacking the obligatory carboxamide tethering 'scar' characteristic for the initial series of scaffolds [9]. Other examples describing the use of branched synthesis strategies as an efficient way to generate scaffold diversity from common synthons have been reported [10].

Another synthetic strategy that appears attractive in this context is the 'libraries from libraries' concept, first advocated by the Houghten group [11][12] (Scheme 2). Therein, a first library produced on solid support is cleaved off only in part, and the remaining resin is subsequently subjected to further chemical transformations to produce secondary libraries, thus again maximizing the return upon investment in terms of chemistry development.

In addition to these strategic considerations, there are also a few synthetic methods that appear superior to others with respect to their versatility and efficiency, in that they can be used to generate a wide range of
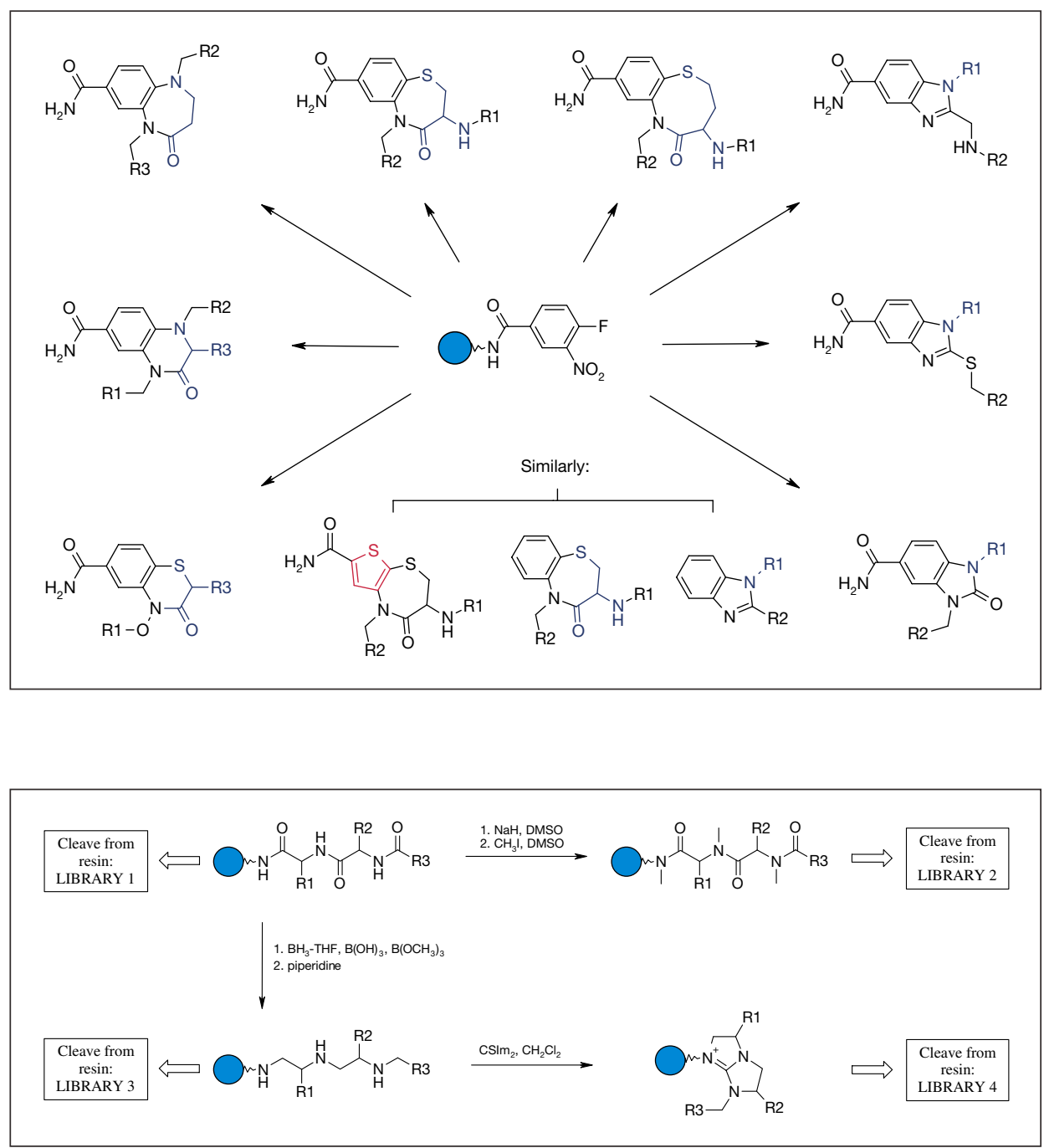

Scheme 1. Maximizing the return upon investment: versatile chemical synthons and branching synthesis strategies.
Scheme 2. Maximizing the return upon investment: 'libraries from libraries'. 
different chemotypes by simply changing the nature of the reagents, thus typically with a minimal investment in terms of chemistry validation and optimization. A prime example are the multi-component reactions (MCRs), and in view of the evidence presented in this report, it does not come as a surprise that they have re-gained increasing attention in recent years [13], in particular amongst the combinatorial community.

\section{Conclusion}

Towards the end of the past decade, there has been a paradigm shift in the way that the pharmaceutical industry has applied combinatorial chemistry to drug discovery. Primary library synthesis has steered away from numerically large libraries ( $>10,000$ members) of a limited selection of chemotypes, and moved towards the synthesis of numerous libraries of moderate size (500-5000 members) comprising many different template structures. The main reason for this change is likely to be found in the disappointing screening results obtained from the first-generation libraries on a variety of biological targets. Using a newly developed computational method to assess the diversity in molecular shape associated with different compound sets (termed NPR-analysis), we have shown that the molecular shape range accessible to the compounds of a given combinatorial library is defined mainly by the central scaffold, whilst the number and nature of the peripheral substituents are of minor importance. Consequently, single-scaffold libraries, irrespective of their size, are restricted to a limited range of molecular shapes, whereas collections of several small libraries around distinct chemical scaffolds produce a higher degree of shape diversity. A comparison of the molecular shape distribution patterns associated with different MDDR subsets of known biological activities further corroborates the intuitive notion that molecular shape is intimately linked to biological activity, and that a high degree of shape (hence scaffold) diversity in screening collections will increase the odds of addressing a broad range of biological targets. In order to cope with the challenge of assembling a portfolio of small libraries around various central scaffolds in a reasonable amount of time and with limited resources, the combinatorial chemist must now, more than ever, seek to optimize the synthetic outcome with respect to the efforts in terms of chemistry set-up and validation. To achieve this goal, it may be advisable to give preference to synthetic strategies and methods that are conducive to the production of a variety of different chemotypes with minimal investment on repeated chemistry validation.

Received: March 23, 2003

[1] A.R. Katritzky, J.S. Kiely, N. Hebert, C. Chassaing, J. Comb. Chem. 2000, $2,2$.

[2] L. Weber, Curr. Opin. Chem. Biol. 2000, 4, 295.

[3] W.H.B. Sauer, M.K. Schwarz, J. Chem. Inf. Comp. Sci. 2003, ACS ASAP (http:// dx.doi.org/10.1021.ci025599w).

[4] L. Xue, J. Bajorath, Comb. Chem. High Throughput Screen. 2000, 3, 363.

[5] J. Gasteiger, C. Rudolph, J. Sadowski, Tetrahedron Comput. Methodol. 1990, 3, 537.

[6] J. Sadowski, C. Rudolph, J. Gasteiger, Anal. Chim. Acta 1992, 265, 233.

[7] M. Brüstle, B. Beck, T. Schindler, W. King, T. Mitchell, T. Clark, J. Med. Chem. 2002, 45, 3345.

[8] E. Fischer, Ber. Dt. Chem. Ges. 1894, 27, 2984.

[9] M.K. Schwarz, M.A. Gallop, in 'SolidPhase Organic Synthesis', Ed. K. Burgess, John Wiley \& Sons, New York, Chichester, Weinheim, Brisbane, Singapore, Toronto, 2000, p. 81 (and references therein).

[10] G.C. Micalizio, S.L. Schreiber, Angew. Chem. Int. Ed. 2002, 41, 3272.

[11] J.M. Ostresh, G.M. Husar, S.E. Blondelle, B. Dörner, P.A. Weber, R.A. Houghten, Proc. Natl. Sci. USA 1994, 91, 11138.

[12] J.M. Ostresh, C.C. Schoner, V.T. Hamashin, A. Nefzi, J-P. Meyer, R.A. Houghten, J. Org. Chem. 1998, 63, 8622.

[13] A. Dömling, Y. Ugi, Angew. Chem. Int. Ed. 2000, 39, 3168 (and references therein). 